\title{
Improving Survival Besides the High Early Mortality Rate in Acute Promyelocytic Leukemia
}

\author{
Umit Yavuz MALKAN ${ }^{1}$, Ebru KOCA ${ }^{2}$, Yahya BUYUKASIK ${ }^{1}$ \\ ${ }^{1}$ Hacettepe University, Faculty of Medicine, Department of Hematology \\ ${ }^{2}$ Baskent University, Faculty of Medicine, Department of Hematology, Ankara, TURKEY
}

\begin{abstract}
The improvement in survivals of acute promyelocytic leukemia (APL) patients are still debated. In this study, we aimed to analyze the survival and early mortality (EM) rates of APL patients. In this study, de-novo APL patients who was followed up by our clinic between the years 2003-2021, were retrospectively analyzed. Patients were divided in according to their year of diagnosis, 2003-2013 ( $n=33$ ) and 2014-2021 ( $n=19$ ) groups. The 2-years survival is 52.5\% and 78.9\% in 2003-2013 group and 2014-2021 group, respectively, $p=$ 0.069.The EM was not statistically different between the 2003-2013 and 2014-2021 groups (11/22 vs.4/15, $p=0.347)$. The 2-years survival ratio is $75.3 \%$ and $36.4 \%$ in modified-AIDA and IDA-ARA/C-ATRA treatment groups, respectively, $p=0.003$. EM according to treatment groups were $7 / 38$ versus $7 / 11$ in modified-AIDA and IDA-ARA/C-ATRA,respectively, $p=0.001$. White blood cell count $\geq$ $10000 / \mu \mathrm{l}$ is the most important predictor of EM with overall prediction of $79.5 \%$. The second most important factor which is related with EM is infection of patients at presentation. If these two factors are considered together there is an $84.1 \%$ overall prediction of EM. The third and last significant factors that effects EM is the induction treatment protocol. If these three significant parameters considered all together,there would be an $88.6 \%$ overall prediction of EM. The overall survival of APL patients is slightly improved between the years 2014-2021 compared to 2003-2013. The reason of this improvement is not the reduction of EM in the APL patients. Better diagnostic tools and molecular monitoring and better supportive care may have played role in the improvement of the survival of APL patients. In order to further improvement of the overall survival of APL patients, EM rates must be decreased. According to our study results, rapid and efficient treatment of infections in APL patients look like the only modifiable parameter that is related with EM.
\end{abstract}

Keywords: Acute promyelocytic leukemia, Survival, Early mortality

\section{INTRODUCTION}

Acute promyelocytic leukemia (APL) is currently classified as acute promyelocytic leukemia with $\mathrm{t}(15 ; 17)(\mathrm{q} 24.1 ; \mathrm{q} 21.2)$; PML-RARA in the World Health Organization classification system. ${ }^{1}$ APL is a distinctive syndrome that should be suspected in patients who present with bleeding or bruising, leukemic blasts with coarse or dense cytoplasmic granules, and/or few circulating leukemic cells. APL is a medical emergency that requires distinctive and urgent treatment. If not treated, APL has a very short median survival of less than one month due to uncontrolled bleeding. APL represents a medical emergency with a high rate of early mortality, often due to hemorrhage from a characteristic coagulopathy. ${ }^{2}$ The incidence of early hemorrhagic death was $3.7 \%$ among nearly 1000 cases that registered on prospective clinical trials which included early administration of all-trans retinoic acid (ATRA). ${ }^{3}$ On the other hand, with current treatment agent including all-trans retinoic acid (ATRA), APL is associated with the highest percentage of patients who are probably cured of their disease. 
However, there is heterogeneity in APL patients. Patients who have a white blood cell (WBC) count less than $10.000 / \mu \mathrm{L}$ found to have longer eventfree survival. ${ }^{4}$ Interestingly, age over 60 years is not a poor prognosis indicator in APL. ${ }^{5}$ Recently published real-life data of APL patients showed that early deaths are still a problem in APL despite the improvement of treatment approaches. ${ }^{6}$ In another recent study it was suggested that the longterm survival remains low in APL patients, particularly related to a high ED rate. ${ }^{7}$ Population-based surveys that included less highly selected APL cases resulted with an early death rate of $17-29 \%{ }^{8,9} \mathrm{~A}$ study that was published in year 2012 stated that the treatment advances have not improved the early death rate in acute promyelocytic leukemia. ${ }^{9}$

In the study, we have aimed to analyze the survival and early death rates of APL patients ranging between the years 2003-2021 and understand the factors that is related with the survival rates.

\section{PATIENTS AND METHODS}

Fifty-two de-novo APL patients who was followed up by our clinic between the years 2003-2021, were retrospectively analyzed. The inclusion criteria of the patients were to be at age $\geq 18$ years at the time of diagnosis and to have APL diagnosis. The APL diagnoses are made according to the World Health Organization criteria. If available, fluorescence in situ hybridization or reverse transcriptionpolymerase chain reaction test was performed to detect translocation (15:17). Also, flow cytometric analyses were used to detect the leukemic blast cells. ED was defined as mortality due to any cause within 30 days after the APL diagnosis. The methods of diagnosis, first application date, diagnosis date, last follow-up date, mortality date, remission date, treatment induction date, age, gender, type of APL, clinical infection/DIC/bleeding at presentation, treatment protocol, treatment results, hemoglobin, white blood cell, platelet, creatinine, fibrinogen, CRP, LDH, Sans score, ECOG and cause of mortality were noted. The modified AIDA regimen was the combination of oral ATRA $\left(45 \mathrm{mg} / \mathrm{m}^{2} /\right.$ day $)$ and idarubicin $\left(12 \mathrm{mg} / \mathrm{m}^{2} /\right.$ day $)$.

Data of the patients were obtained from the hospital database. All of the ethical considerations was strictly followed in accordance with the 1964 Helsinki declaration. As a standard care/action of the hospitals of the Hacettepe University Hospitals, it has been recognized from the patient records that all of the studied patients had given informed consents at the time of hospitalization and before the administration of chemotherapy and other relevant diagnostic/therapeutic standard of care.

\section{Statistical Analysis}

Statistical analyses were done by the SPSS software v.25. The APL patients are divided into two major groups according to their diagnosis periods which are the years of 2003-2013 and 2014-2021. The categorical parameters of these two groups are compared with chi-square test or Fisher's exact test. The continuous variables are tested for normality with Kolmogorov-Smirnov test. Parametric continuous variables are compared with T-test, whereas the non-parametric continuous variables are compared with Mann-Whitney U test.

The primary endpoint of the study was defined as the overall survival durations of the APL patients. The secondary endpoint of the study is the early death rates. The third endpoint of the study is to analyze the parameters which is related with survival and death rates. Univariate analysis of parameters related with OS are calculated with Kaplan-Meier method. Parameters that were significant by the mean of $p$ value $<0.1$, were taken to the next step. Multivariate analysis of parameters related with OS are calculated with Cox-Regression analysis forward like-hood ratio method.

In order to understand the parameters related with $\mathrm{ED}$, univariate binary logistic regression was performed to each parameter. Parameters that were significant by the mean of $\mathrm{p}$ value $<0.1$, were taken to the next step. Multivariate logistic regression analysis with forward like-hood ratio method was performed to understand the parameters that are related with ED.

\section{RESULTS}

33 and 19 patients were included in the 2003-2013 and 2014-2021 groups, respectively. 22/33 and $17 / 19$ patients were diagnosed with reverse tran- 


\begin{tabular}{|c|c|c|c|}
\hline Parameter & $2003-2013(n=33)$ & $2014-2021(n=19)$ & $P$ value \\
\hline Gender (F/M) & $20 / 13$ & $9 / 10$ & 0.355 \\
\hline Age (years) & $42(17-63)$ & $49(24-68)$ & 0.050 \\
\hline Hemoglobin (gr/dl) & $9.1(4.0-13.4)$ & $9.8(6.0-15.6)$ & 0.309 \\
\hline White blood cell $\left(x 10^{\wedge} 3 / \mu \mathrm{L}\right)$ & $2.6(0.5-155.7)$ & $2.2(0.3-30.5)$ & 0.739 \\
\hline 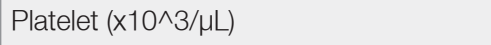 & $19(5-83)$ & $29(13-153)$ & 0.500 \\
\hline Albumin (gr/dl) & $4.3(2-5)$ & $4.3(3-4.9)$ & 0.787 \\
\hline Creatinine (mg/dl) & $0.77(0.43-1.67)$ & $0.81(0.5-2.3)$ & 0.367 \\
\hline Fibrinogen (mg/dl) & $169(97-464)$ & $164(35-525)$ & 0.575 \\
\hline C-reactive protein (mg/dl) & $3.2(0.5-24.8)$ & $2.3(0.3-45.3)$ & 0.286 \\
\hline Lactate dehydrogenase (U/L) & $560(201-7222)$ & $312(108-493)$ & 0.001 \\
\hline Induction Result (CR/Other) & $21 / 30$ & $15 / 19$ & 0.446 \\
\hline Ex Status & $16 / 33$ & $4 / 19$ & 0.050 \\
\hline Bleeding at presentation & $6 / 33$ & $2 / 19$ & 0.461 \\
\hline $\begin{array}{l}\text { Disseminated intravascular coagulation } \\
\text { at presentation }\end{array}$ & $13 / 33$ & $7 / 19$ & 0.855 \\
\hline Infection at presentation & 20/33 & $9 / 19$ & 0.355 \\
\hline Microvariant type leukemia & $5 / 33$ & 0/19 & 0.126 \\
\hline $\begin{array}{l}\text { Modified AIDA / Idarubisin- cytosine } \\
\text { arabinoside - ATRA }\end{array}$ & $23 / 7$ & $15 / 4$ & 0.852 \\
\hline Sanz Score (low/intermediate/high) & $11 / 12 / 10$ & $7 / 9 / 3$ & 0.492 \\
\hline ECOG (0/1/2/3/4) & $9 / 17 / 3 / 2 / 2$ & $5 / 11 / 3 / 0 / 0$ & 0.580 \\
\hline Early Mortality (Y/N) & $11 / 22$ & $4 / 15$ & 0.347 \\
\hline
\end{tabular}

scriptase polymerase chain reaction test (RT-PCR) or conventional cytogenetic (CC) in each groups, $\mathrm{p}: 0.102$. The other parameters of the patients were given in Table 1. Three patients could not be given induction therapy because of early mortality. The continuous variables were tested for normality (Table 2). The parameters of two groups were similar except the serum Lactate dehydrogenase levels.

There is a borderline statistically significant difference between the overall survival of the two groups. The 2-years survival ratio is $52.5 \%$ and $78.9 \%$ in 2003-2013 groups and 2014-2021 groups, respectively, $p=0.069$. Among the patients who were given induction therapy, the modified AIDA treatment has better results than idarubisin- cytosine arabinoside - ATRA combination. The 2-years survival ratio is $75.3 \%$ and $36.4 \%$ in modified AIDA and IDA-ARA/C-ATRA groups, respectively, $\mathrm{p}=0.003$. Moreover, the early mortality is more frequent in IDA-ARA/C-ATRA group. Among 38 patients who were given modi- fied AIDA protocol, there were 7 early mortalities; whereas among 11 patients who were given IDAARA/C-ATRA protocol, there were 7 early mortalities, also, which is statistically significant, $\mathrm{p}=$ 0.001 . There was a statistically significant different between the treatment choices and Sanz score of the study patients. There were 14 low risk, 19 intermediate risk, and 5 high risk patients versus 3 low risk, 1 intermediate risk, and 7 high risk patients in modified AIDA groups and idarubisin-cytosine arabinoside-ATRA groups respectively, $\mathrm{p}=0.002$. According to the white blood count and treatment choices, patients also have statistically significant difference. Patients were given 5 modified AIDA and 7 idarubisin-cytosine arabinoside-ATRA versus 33 modified AIDA and 4 idarubisin-cytosine arabinoside-ATRA protocols in white blood cell $10.000 / \mu \mathrm{L}$ or higher and white blood cell lower than $10000 / \mu \mathrm{L}$ groups, respectively, $\mathrm{p}=0.001$.

There were a total of 20 deaths in our study group. Infection was the most frequent mortality reason 


\begin{tabular}{|ll|}
\hline Table 2. Test of Normality & \\
\hline Parameter & $\begin{array}{l}\text { Kolmogorov-Smirnov } \\
\text { Significance }\end{array}$ \\
\hline Age & 0.2 \\
Hemoglobin & 0.2 \\
White Blood Cell & $<0.001$ \\
Platelet & $<0.001$ \\
Albumine & 0.052 \\
Creatinine & $<0.001$ \\
Fibrinogen & 0.033 \\
CRP & $<0.001$ \\
LDH & $<0.001$ \\
\hline
\end{tabular}

$(13 / 20)$ whereas bleeding $(6 / 20)$ is the second frequent cause of mortality among our study participants. One patient had died from a secondary malignancy. There were 15 early deaths among the total of 20 deaths in our study, indicating that the three quarter of all death were in the first 30 days from the diagnosis of APL. The reasons of mortality are slightly different between patients. The reasons of mortality were 9 infections, 6 bleeding and 0 other causes compared to 4 infections, 0 bleeding and 1 other causes in the early mortality versus non-early mortality patients, respectively, with a borderline statistical significance; $\mathrm{p}=0.073$.

We have aimed to investigated the early mortality because the early mortality among our study group was found to be related with the overall survival of the patients $(p<0.001)$. The early mortality was not statistically different between the 2003-2013 and 2014-2021 groups (11/22 vs. 4/15, p:0.347). The parameters that can be related to early mortality is also analyzed with logistic regression analysis. The parameters with $\mathrm{p}<0.05$ in univariate logistic regression analysis (Table 3), were analyzed with multivariate logistic regression analysis with forward like-hood method. The white blood cell count, infection at presentation and induction treatment protocol is found to be related with early mortality of APL patients (Table 4). According to this method, white blood cell count 10000 or higher is the most important predictor of early mortality with overall prediction percentage of $79.5 \%$. The second most important factor which is related with

\begin{tabular}{|c|c|}
\hline Parameter & $P$ value \\
\hline Gender & 0.696 \\
\hline Age & 0.894 \\
\hline White blood cell count $10000 / \mu \mathrm{L}$ or higher & 0.001 \\
\hline Hemoglobin $10 \mathrm{gr} / \mathrm{dl}$ or higher & 0.153 \\
\hline Platelet 40000 or higher & 0.205 \\
\hline Lactate dehydrogenase $250 \mathrm{U} / \mathrm{L}$ or higher & 0.316 \\
\hline Fibrinogen 150 mg/dl or lower & 0.026 \\
\hline CRP 5 mg/dl or higher & 0.028 \\
\hline Creatinine $1 \mathrm{mg} / \mathrm{dl}$ or higher & 0.794 \\
\hline Albumin $4 \mathrm{gr} / \mathrm{dl}$ or lower & 0.380 \\
\hline ECOG score & 0.006 \\
\hline Sanz score & 0.006 \\
\hline Bleeding at presentation & 0.006 \\
\hline $\begin{array}{l}\text { Disseminated intravascular coagulation } \\
\text { at presentation }\end{array}$ & 0.011 \\
\hline Infection at presentation & 0.003 \\
\hline Induction treatment protocol & 0.002 \\
\hline Diagnosis year 2003-2013 or 2014-2021 & 0.350 \\
\hline
\end{tabular}

early mortality is infection of patients at presentation. If these two factors are considered together there is an $84.1 \%$ overall prediction percentage of early mortality. The third and last significant factors that effects early mortality is the induction treatment protocol. If these three significant parameters considered all together, there would be an $88.6 \%$ overall prediction percentage of early mortality.

\section{DISCUSSION}

In the literature there are previous studies that focus on the survival rates of APL patients. It has been stated that over the past decades, a remarkable advance has been seen in survival of APL, especially after the inclusion of ATRA. ${ }^{6}$ A previous study by Akcay et al. reported that long-term survival remains low in APL patients, mostly associated with a high early death rate. ${ }^{7}$ Authors have stated that clinical awareness, rapid confirmation of the diagnosis, and quick administration of ATRA is the key elements to avoid early death in APL patients. Similarly, in our study three quarter of all death were in the first 30 days from the diagnosis of APL. 
International Journal of Hematology and Oncology

Table 4. Multivariate logistic regression analysis of parameters related with early mortality

\begin{tabular}{|llllr}
\hline Parameter & Beta & P value & Exp (B) & \multicolumn{2}{c}{ 95\% C.I.for EXP(B) } \\
& & & & Lower \\
Upper
\end{tabular}

Recently, induction of ATRA and ATO, better diagnostic tools and molecular monitoring, better supportive care enabled better results in APL patients. ${ }^{10}$ In our study we have evaluated the patients in two groups according to their diagnosis year which are the 2003-2013 and 2014-2021 groups. The all parameters between the two groups were same except the serum lactate dehydrogenase levels. According to our results, RT-PCR and CC test are more frequently used in 2014-2021 group. This indicates the laboratory diagnostic test techniques are improving over the time and clinician use the supporting laboratory test more frequently. Moreover, in our study the 2-years survival ratio is $\% 52.5$ and $78.9 \%$ in 2003-2013 groups and 2014-2021 groups, respectively, $\mathrm{p}=0.069$. This results indicates borderline statistical significance between the two groups, indicating the prognosis of APL patients are getting better despite the high early mortality rates. The importance of quicker diagnosis and the need for a better supportive care in APL patients was mentioned in previous studies nearly a decade ago. ${ }^{11}$ The results in our study may indicate that better diagnostic tools and molecular monitoring and better supportive care may have played a role in the long-term survival of APL patients in the last decade. Also, accumulated experience with APL patients may also lead to better management of the adverse effects of APL treatment agents, such as ATRA. ${ }^{12}$

In our study we have found that the white blood cell count, infection at presentation and induction treatment protocol is found to be related with early mortality of APL patients. In the literature the Sanz score is well-defined risk factor for APL. ${ }^{13}$ Similarly, in our study white blood cell count 10000 or higher is the most important predictor of early mor- tality with overall prediction percentage of $79.5 \%$. On the other, in our study hand platelet levels are not found to be related with early mortality.

In previous studies, the relation between coagulopathy and early death is reported in APL patients. ${ }^{14}$ On the other hand, in a study by Silva et al. bleeding events were found to be the main cause of mortality before receiving chemotherapy in APL patients. ${ }^{6}$ In our study the most frequent reason of death was infections however, mortality from bleeding is distinctly higher in early mortality patients. Moreover, in our patient the second most important factor which is related with early mortality is infection of patients at presentation. According to our results, if white blood cell count and infection status of APL patients are considered together there is an $84.1 \%$ overall prediction percentage of early mortality.

In the literature, it was stated that daunorubicin, idarubicin and cytosine arabinoside was the frontline treatment of APL with a complete remission rate of $75 \%$ to $80 \%$ in newly diagnosed patients. ${ }^{10}$ However, it is well-known that All-trans-retinoic acid as induction or maintenance treatment improves disease-free and overall survival as compared with chemotherapy alone. ${ }^{15}$ The PETHEMA group reported that combining anthracycline and ATRA for induction and consolidation therapy of newly diagnosed APL results in improved anti-leukemic efficacy. ${ }^{16}$ German AMLCG groups reported that ATRA and intensified chemotherapy including high dose cytosine arabinoside reduced the relapse risk in high risk APL patients. ${ }^{17}$ Moreover previous studies reported that anthracycline-based treatment is at least equally effective as cytarabine-containing regimens for low-/intermediate-risk APL patients and need of cytarabine coupled to anthracyclines and ATRA during consolidation in the high-risk 
group. ${ }^{18}$ According with the literature, in our study high risk group patients according to their Sanz score were given idarubisin-cytosine arabinosideATRA protocol more frequently. Moreover, in our study, patients who have white blood cell count $10000 / \mu \mathrm{L}$ or more were given idarubisin-cytosine arabinoside-ATRA protocol more frequently, also. In our study the third most important parameter that is related with early mortality was found to be the induction treatment protocols. The reason of this can be the physician choices idarubisin-cytosine arabinoside-ATRA protocol to poor prognostic APL patients in our study. If these white blood cell count, infection status and treatment choices of the patients considered all together, there would be an $88.6 \%$ overall prediction percentage of early mortality according to the results of our study.

Our study is limited by its retrospective design and the limited number of study participants. However, although it has some limitations, this study may reflect the recent survival improvement in the APL patients despite the relatively high early death rates.

\section{CONCLUSION}

In conclusion, the overall survival of APL patients is slightly improved between the years 2014-2021 compared to 2003-2013. The reason of this improvement is not the reduction of early mortality in the APL patients. better diagnostic tools and molecular monitoring and better supportive care may have played a role in the improvement of the survival of APL patients. In our study we have found that the white blood cell count, infection at presentation and induction treatment protocol is found to be related with early mortality of APL patients. In order to further improvement of the overall survival of APL patients, early mortality rates must be decreased. According to our study results, in order to control the early mortality rates of APL patients, rapid and efficient treatment of infections in APL patients look like the only modifiable parameter that is related with early mortality. There is a need for future prospective randomized trials in order to deal with the unmet need in APL patients which is the early mortality rates.

\section{REFERENCES}

1. Arber DA, Orazi A, Hasserjian R, et al. The 2016 revision to the World Health Organization classification of myeloid neoplasms and acute leukemia. Blood 127: 2391-2405, 2016.

2. Park JH, Qiao B, Panageas KS, et al. Early death rate in acute promyelocytic leukemia remains high despite all-trans retinoic acid. Blood 118: 1248-1254, 2011.

3. Mantha S, Goldman DA, Devlin SM, et al. Determinants of fatal bleeding during induction therapy for acute promyelocytic leukemia in the ATRA era. Blood 129: 1763-1767, 2017.

4. Asou N, Adachi K, Tamura J, et al. Analysis of prognostic factors in newly diagnosed acute promyelocytic leukemia treated with all-trans retinoic acid and chemotherapy. Japan Adult Leukemia Study Group. J Clin Oncol 16: 78-85, 1998.

5. Latagliata R, Avvisati G, Lo Coco F, et al. The role of all-transretinoic acid (ATRA) treatment in newly-diagnosed acute promyelocytic leukemia patients aged $>60$ years. Ann Oncol 8: 1273-1275, 1997.

6. Silva WFD, Jr., Rosa LID, Marquez GL, et al. Real-life outcomes on acute promyelocytic leukemia in Brazil - Early deaths are still a problem. Clin Lymphoma Myeloma Leuk 19: 116-122, 2019.

7. Akcay OF, Yeter HH, Buyukasik Y. Real-life outcomes of unselected acute promyelocytic leukemia patients: a singlecenter 14-year experience. Rom J Intern Med 58: 138-145, 2020.

8. Lehmann S, Ravn A, Carlsson L, et al. Continuing high early death rate in acute promyelocytic leukemia: a populationbased report from the Swedish Adult Acute Leukemia Registry. Leukemia 25: 1128-1134, 2011.

9. McClellan JS, Kohrt HE, Coutre S, et al. Treatment advances have not improved the early death rate in acute promyelocytic leukemia. Haematologica 97: 133-136, 2012.

10. Wang ZY, Chen Z. Acute promyelocytic leukemia: from highly fatal to highly curable. Blood 111: 2505-2515, 2008.

11. Serefhanoglu S, Buyukasik $\mathrm{Y}$, Goker $\mathrm{H}$, et al. Clinical features and outcomes of 49 Turkish patients with acute promyelocytic leukemia who received ATRA and anthracyclines (PETHEMA protocol) therapy. Leuk Res 34: 317-319, 2010.

12. Afacan Ozturk HB, Albayrak M, Maral S, et al. Hypercalcemia associated with the interaction between all trans retinoic acid and posaconazole in an acute promyelocytic leukemia case. J Oncol Pharm Pract 2021: 10781552211007889, 2021.

13. Sanz MA, Lo Coco F, Martín G, et al. Definition of relapse risk and role of nonanthracycline drugs for consolidation in patients with acute promyelocytic leukemia: a joint study of the PETHEMA and GIMEMA cooperative groups. Blood 96: 1247-1253, 2000

14. Kwaan $\mathrm{HC}$. The unique hemostatic dysfunction in acute promyelocytic leukemia. Semin Thromb Hemost 40: 332-336, 2014. 
15. Tallman MS, Andersen JW, Schiffer CA, et al. All-trans-retinoic acid in acute promyelocytic leukemia. N Engl J Med 337: 1021-1028, 1997.

16. Sanz MA, Martín G, González M, et al. Risk-adapted treatment of acute promyelocytic leukemia with all-trans-retinoic acid and anthracycline monochemotherapy: a multicenter study by the PETHEMA group. Blood 103: 1237-1243, 2004.

17. Lengfelder E, Haferlach C, Saussele S, et al. High dose ara-C in the treatment of newly diagnosed acute promyelocytic leukemia: long-term results of the German AMLCG. Leukemia 23: 2248-2258, 2009.

18. Lo-Coco F, Avvisati G, Vignetti M, et al. Front-line treatment of acute promyelocytic leukemia with AIDA induction followed by risk-adapted consolidation for adults younger than 61 years: results of the AIDA-2000 trial of the GIMEMA Group. Blood 116: 3171-3179, 2010.

\section{Correspondence:}

\section{Dr. Umit Yavuz MALKAN}

Hacettepe Üniversitesi Tip Fakultesi

Hematoloji Bilim Dali

06230 Sihhiye

ANKARA / TURKEY

Tel: (+90-532) 7780087

e-mail: umitmalkan@hotmail.com

\section{ORCIDs:}

Umit Yavuz Malkan

Ebru Koca

Yahya Buyukasik
0000-0001-5444-4895

0000-0002-7566-4456

0000-0002-4764-2348 